\title{
Intestinal barrier loss as a critical pathogenic link between inflammatory bowel disease and graft-versus-host disease
}

\author{
SC Nalle ${ }^{1,2}$ and JR Turner ${ }^{2}$
}

Compromised intestinal barrier function is a prominent feature of inflammatory bowel disease (IBD). However, links between intestinal barrier loss and disease extend much further, including documented associations with celiac disease, type I diabetes, rheumatoid arthritis, and multiple sclerosis. Intestinal barrier loss has also been proposed to have a critical role in the pathogenesis of graft-versus-host disease (GVHD), a serious, potentially fatal consequence of hematopoietic stem cell transplantation. Experimental evidence has begun to support this view, as barrier loss and its role in initiating and establishing a pathogenic inflammatory cycle in GVHD is emerging. Here we discuss similarities between IBD and GVHD, mechanisms of intestinal barrier loss in these diseases, and the crosstalk between barrier loss and the immune system, with a special focus on natural killer (NK) cells. Unanswered questions and future research directions on the topic are discussed along with implications for treatment.

\section{INTRODUCTION}

The intestinal barrier includes extracellular components, such as mucin, but ultimately depends on the presence of a continuous epithelial monolayer. This article therefore focuses primarily on the epithelial barrier; related topics, including mucosal immunity, extracellular mucin barriers, and the microbiome have recently been reviewed in refs. 1-9 and are not considered further here.

Epithelial barrier loss can occur as a result of direct epithelial cell damage or through more subtle changes in paracellular tight junction permeability. These forms of intestinal barrier loss, when dysregulated, are thought to contribute to the initiation and propagation of the inflammatory bowel diseases (IBD), Crohn's disease and ulcerative colitis. ${ }^{10,11}$ Graft-versushost disease (GVHD), which develops after bone marrow transplantation (BMT) or, more recently, hematopoietic stem cell transplantation, shares genetic associations and some clinical manifestations with IBD. ${ }^{12-14}$ However, despite abundant correlative reports, it is only recently that direct evidence for intestinal barrier loss as a driving mechanism in GVHD, as well as IBD, has become available. ${ }^{15-18}$ This barrier loss may contribute to or work in concert with alterations in the gut microbiome in GVHD and IBD. ${ }^{19-23}$ What is perhaps most striking about the loss of both barrier function and microbial diversity in GVHD is that, in addition to amplifying intestinal disease and reducing survival, these factors impact disease in other target organs, including the liver, skin, and lungs. Here, we review current understanding of intestinal epithelial barrier loss and its contributions to the pathogenesis of immune-mediated disease and address critical unanswered questions.

\section{IBD AND GVHD: THE SAME, BUT DIFFERENT?}

The similarities between IBD and GVHD extend beyond the presence of epithelial barrier defects: there is also a significant overlap in clinical and pathological manifestations of these diseases (Table 1). For example, symptoms of both IBD and GVHD can include abdominal pain, nausea, malabsorption, diarrhea, and, likely as a result of these, weight loss. Notably, the nature of the diarrhea differs, as it is often bloody or mucoid in IBD, but more watery in GVHD. This reflects marked differences in mechanism and extent of tissue damage, which is clearly demonstrated by the intestinal histopathology of these diseases. GVHD is characterized by crypt-cell apoptosis and 
Table 1 Similarities and differences between IBD and GVHD

\begin{tabular}{lll}
\hline & Similarities & Differences \\
\hline $\begin{array}{ll}\text { Genetic associations at } \\
\text { microbial-sensing loci }\end{array}$ & Polymorphisms of TLR-4, TLR-5 and TLR-9; NOD-2; & Polymorphisms of TLR2 and TLR6 (IBD) \\
Immunological aspects & ATG16L1 & \\
& Tissue and serum TNF correlate with severity of clinical Neutrophilic (IBD) & Highly T cell mediated (GVHD) \\
& and experimental disease & Antigens are undefined in IBD, but relatively-defined in GVHD \\
& IFN- $\gamma$ and IL-1 $\beta$ are elevated in patients and experi- & Anti-TNFbiologics are highly effective in IBD (particularly Crohn's \\
& mental models & disease) and experimental models of IBD and GVHD, but are not \\
& Immunosuppressants (e.g., corticosteroids) and & immomodulators (e.g., methotrexate) are frequently yet defined in human GVHD \\
& helpful in treating disease &
\end{tabular}

Barrier dysfunction

Gut microbiota
Correlates with disease severity in patients and experimental models

Required for disease in MHC-matched GVHD experimental model

Proinflammatory milieu modulates tight junction components

Required for disease in most experimental models Loss of microbial diversity observed in humans

Extraintestinal manifestations Portal inflammation and bile duct damage in liver Skin involvement
Present in some healthy first-degree relative and may predict relapse in patients during remission (IBD)

MLCK inhibition limits disease in experimental models of IBD; has not been reported in GVHD

Antibiotics can be preventative in GVHD patients, use in IBD is controversial

Peribronchialar inflammation and damaged airway epithelium in the lung (GVHD)

Abbreviations: GVHD, graft-versus-host disease; IBD, inflammatory bowel disease; IFN, interferon; IL, interleukin; MHC, major histocompatability complex; MLCK, myosin light chain kinase; TLR, toll-like receptor; TNF, tumor necrosis factor.

glandular atrophy. The immune reaction is often sparse, and both mucosal neutrophil infiltration and ulceration are present in only the most severe cases. In contrast, disease activity in IBD is defined by mucosal and intraepithelial neutrophils that infiltrate and damage the epithelium, forming crypt abscesses. Typically, these are accompanied by dense accumulations of lymphocytes, macrophages, and plasma cells within the lamina propria (Figure 1). Although epithelial apoptosis can be present in IBD, it is not a prominent histologic feature in patient biopsies except when induced by certain therapeutic agents such as mycophenolate. Some experimental models of IBD induce intestinal epithelial apoptosis via high dose cytokine treatment or chemical damage, e.g., with dextran sodium sulfate. These models are most useful in studies of the inflammatory response to epithelial damage rather than the more complex interactions at play in human disease. This is, in part, the reason that many no longer consider dextran sodium sulfate colitis to be as adequate as a model of IBD. ${ }^{24,25}$

Ultimately, both GVHD and IBD can lead to mucosal, i.e., glandular, atrophy. ${ }^{26}$ However, the crypt architectural distortion that results from repeated injury and repair in IBD is not prominent in GVHD. Overall, some of these pathologic differences may be explained by contrasting the cycling between remission and active disease typically observed in IBD patients with the kinetics of GVHD, which begins after transplantation and tends to persist, with variable intensity, for months to years.

Extraintestinal manifestations of IBD and GVHD also display some overlap. Ulcerative colitis can be complicated by primary sclerosing cholangitis, in which there is bile duct loss because of progressive fibrosis, whereas immune-mediated bile duct damage is common in GVHD. Furthermore, both GVHD and Crohn's disease can involve the integument, although the histopathologies are very different.
Finally, some therapeutic agents are useful in both IBD and GVHD, likely reflecting the shared pathogenic mechanisms (Figure 1). These treatments include immunosuppressants and calcineurin inhibitors, e.g., tacrolimus, ${ }^{27-32}$ although the sequence in which specific agents are employed differs between the diseases. In addition, anti-tumor necrosis factor (TNF) biologics, some of which are mainstays of the IBD therapy, ${ }^{33-35}$ have been effective in mouse models of $\mathrm{GVHD}^{36-38}$ and are now being investigated in patients. ${ }^{39-43}$ Furthermore, growth factors, including keratinocyte growth factor (KGF), ${ }^{44-47}$ epidermal growth factor, ${ }^{48,49}$ and R-spondin, ${ }^{50,51}$ which promote mucosal healing and restoration of barrier function, have been shown to be effective in both IBD and GVHD models and are also being evaluated in patients.

\section{Shared microbial-sensing defects}

A likely possibility is that intestinal barrier dysfunction contributes to disease pathogenesis in IBD and GVHD by allowing microbes (in the case of epithelial damage), and microbial products (when paracellular permeability is increased), to cross the barrier and engage pattern-recognition receptors (PRRs) on the basolateral surface of epithelial cells, as well as hematopoietic and non-hematopoietic cells within the mucosa. PRRs recognize structures that are conserved among large groups of microbes. Engagement of these sensors elicits inflammasome activation, pro-inflammatory cytokine release, chemokine secretion, and antigen-presenting cell maturation, thereby enabling an effective immune response. ${ }^{52}$ Conversely, PRRs may also dampen the inflammatory responses to promote resolution or immune tolerance.

In the context of GVHD, preclinical BMT models have demonstrated roles for several PRRs, including toll-like receptor (TLR-4), TLR-5, TLR-9, and the intracellular PRR NOD-2, which recognize lipopolysaccharide (LPS), flagellin, 


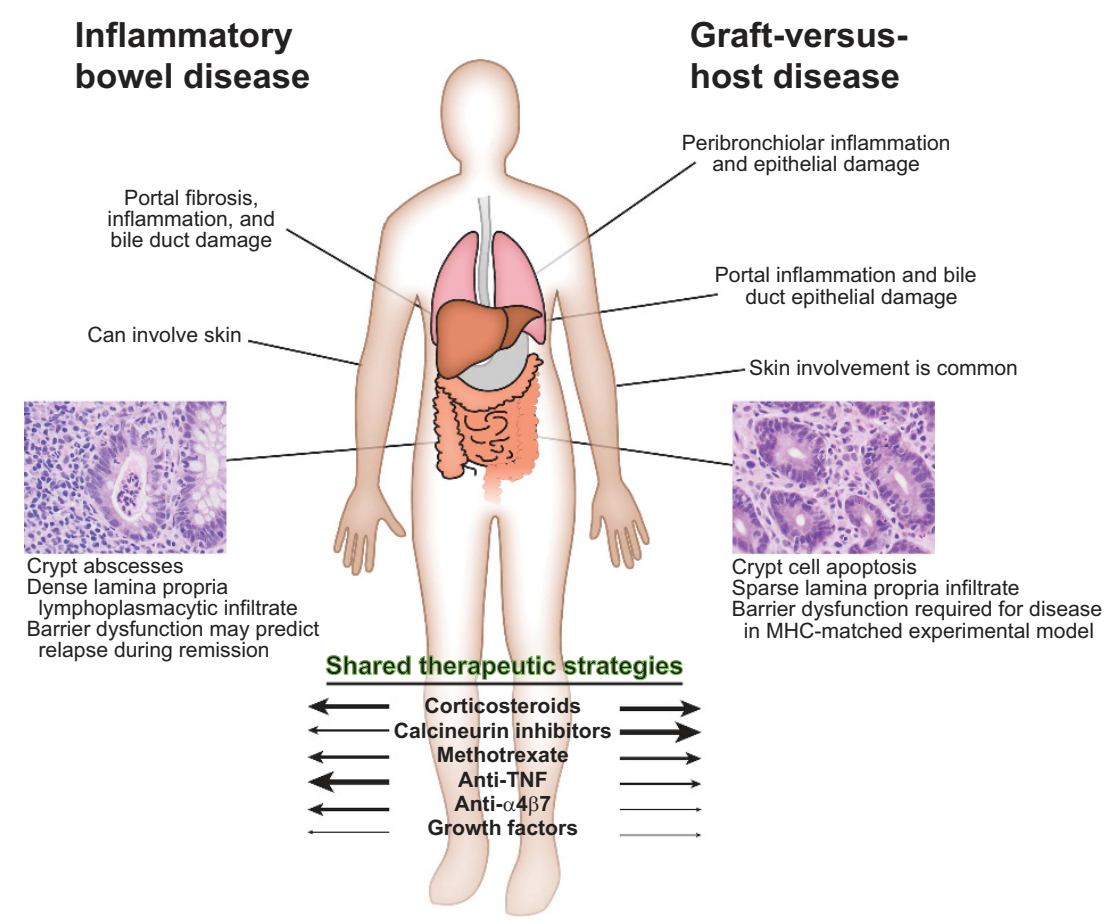

Figure 1 Comparing and contrasting inflammatory bowel disease and graft-versus-host disease. Weight of arrows indicates therapy usage prevalence for each disease.

unmethylated cytosine phosphorothioate-guanine, and muramyl dipeptide, respectively. TLR-4 has been the most wellstudied PRR in GVHD, and either LPS antagonism or donors lacking functional TLR-4 limit the severity of experimental disease ${ }^{53-55}$ Further, TLR-9 knockout in recipients, as well as TLR- 5 blockade both reduce the severity of experimental GVHD. ${ }^{19,56,57}$ Conversely, NOD-2 $2^{-1-}$ mice develop more severe GVHD following BMT, ${ }^{58}$ consistent with the unregulated immune activation in response to inflammation that has been observed in the absence of NOD-2. ${ }^{59,60}$ Correlative patient data are consistent with these data, as TLR-4,TLR-5, and TLR-9 polymorphisms are associated with increased GVHD severity after allogeneic hematopoietic stem cell transplantation. ${ }^{61-64}$ In addition, polymorphisms of $N O D$-2, which are strongly linked to Crohn's disease, ${ }^{65-68}$ may be one of the most reliable genetic risk factors for GVHD. ${ }^{62,69-71}$ In one report, NOD-2 polymorphisms in the patient, donor, or both were strongly associated with more severe GVHD. ${ }^{69}$

A role for TLRs in experimental IBD is supported by studies showing that immune cells lacking MyD88, the intracellular signaling adaptor for all TLRs except TLR-3, fail to cause adoptive transfer colitis. ${ }^{72,73}$ Moreover, MyD88 is required for the development of disease in $I L-10^{-1-}$ mice. ${ }^{74}$ Studies of NOD-2 in IBD models complement the findings in GVHD, as the mice that lack NOD-2 have microbial dysbiosis of the gut, ${ }^{60,75,76}$ impaired tolerance to bacterial stimuli, ${ }^{77}$ defective T-cell migration, ${ }^{78}$ and increased susceptibility to smallintestinal inflammation. ${ }^{59}$ Like GVHD, NOD-2, TLR-4, $T L R-5$, and TLR-9 polymorphisms have all been linked to Crohn's disease and, in some cases, ulcerative colitis. ${ }^{79-81}$
Although not a PRR, ATG16L1, is another locus where polymorphisms are a well-established risk factor for Crohn's disease. ${ }^{82,83}$ Likewise, when investigated in the context of hematopoietic stem cell transplantation, it was found that the T300A ATG16L1 variant increased the risk of GVHD and treatment-related mortality, whether present in the donor, recipient, or both. ${ }^{84}$ Interestingly, a recent study investigating the role of Atg16L1 in the context of allogeneic BMT found that the absence of Atg16L1 in host dendritic cells led to alloreactive T-cell hyperactivation and enhanced GVHD severity. ${ }^{85}$ Overall, it is important to recognize that although compromised function of these gene products is linked to both IBD and GVHD, polymorphisms are thought to be associated with initial disease pathogenesis in the former, but are better considered to be modifiers of antigen mismatch-driven disease severity in the latter.

\section{INTESTINAL BARRIER LOSS IN IBD AND GVHD Views and terms in barrier loss}

The current view of intestinal barrier function considers three non-mutually exclusive permeability routes: the paracellular pore and leak pathways and the unrestricted pathway. The pore pathway is a high capacity, charge- and size-selective route across the tight junction that does not allow macromolecules with a radii greater than $\sim 4 \AA$ to pass. In the intestine, monovalent cations and water are the most common molecules that traverse the pore pathway. The leak pathway, which allows larger molecules, likely up to a radii of $\sim 60 \AA$, to cross the tight junction, is not charge-selective. Notably, this size limit is 
considerably smaller than bacteria and viruses, but may allow their products, e.g., LPS, to cross. The leak pathway does not typically overwhelm the pore pathway because the former is a low-capacity route. ${ }^{86}$ Flux across the unrestricted pathway, which is increased following apoptosis, necroptosis, or cytotoxic epithelial damage, allows massive flux of ions, water, macromolecules, and larger materials, including whole bacteria. An alternate pathway of transport across the epithelium is transcytosis, where macromolecules and other antigens are transported from the apical, i.e., luminal, to the basolateral surface by vesicular traffic. Like paracellular pathways, transcytosis can transport material in both directions; however, transcytosis is an active vectorial process. Transcytosis may be important for $\mathrm{IBD}^{87,88}$ but has not been studied in GVHD. Overall, the regulation of transcytosis is quite different from the pathways discussed here and is likely most relevant in specific epithelial cell types, such as $\mathrm{M}$ cells. ${ }^{89-91}$

The tight junction forms an apical belt-like structure around intestinal epithelial cells ${ }^{92}$ and is the primary determinant of the paracellular pore and leak pathways. Flux through the pore pathway is regulated by the composition and stability of individual tight junction proteins, which in turn respond to the local cytokine environment. For example, interleukin (IL-13) can increase cation flux by upregulating expression of the tight junction pore-forming protein claudin-2. ${ }^{93} \mathrm{IL}-13$ expression is increased in ulcerative colitis and, to a lesser extent, in Crohn's disease. ${ }^{94,95}$ IL-13 upregulation is also a reliable prognostic marker of GVHD. ${ }^{96}$ These observations suggest that the pore pathway may contribute to the pathogenesis of these diseases.

In contrast to IL-13-induced claudin-2 expression-dependent pore pathway regulation, flux across the leak pathway is most often governed by epithelial myosin light chain kinase (MLCK) signaling. This can be activated by a variety of stimuli, including TNF superfamily cytokines. ${ }^{97-100}$ MLCK-dependent MLC phosphorylation triggers occludin removal from the tight junction and is associated with reduced-occludin expression, both in experimental models and human patients. ${ }^{94,97,98,101,102}$ Occludin downregulation has also been reported in a mouse GVHD model, ${ }^{16}$ although the significance of this observation is not clear.

The data above show that pore and leak pathways can be governed independently, and it is common to think of these separately. Nevertheless, there is a substantial overlap in their regulatory mechanisms. For example, ZO-1 and occludin anchoring and protein interactions at the tight junction impact both pore and leak permeability. ${ }^{93,101,103,104}$ Further, transgenic intestinal epithelial expression of constitutively active MLCK triggers increases in mucosal IL-13 expression, epithelial claudin-2 expression, and pore pathway permeability. ${ }^{93} \mathrm{Con}-$ versely, colitis-associated claudin-2 expression is reduced in knockout mice lacking the long MLCK isoform expressed in the intestinal epithelia. ${ }^{105}$ Thus, in addition to shared regulatory mechanisms, there is crosstalk between pore and leak pathways.

The unrestricted pathway allows flux of nearly all luminal contents as a result of the epithelial damage. Because tight junctions are absent in areas of epithelial loss, the unrestricted pathway is, by definition, tight junction independent. A previous, less nuanced view of intestinal barrier loss depicted a binary model in which there was increased flux along the unrestricted pathway or, alternatively, barrier function was intact. As appreciation and understanding of the differential regulation of epithelial paracellular permeability on the basis of size and charge selectivity grew, the model was adjusted to include tight junction-mediated pore and leak pathways, as well as the unrestricted pathway. However, incorrect attribution of increased permeability to a particular pathway still occurs. For example, increases in permeability to macromolecular probes, such as the leak and unrestricted pathway probe FITC-4kD dextran, are often attributed to increased epithelial claudin-2 expression, ${ }^{106,107}$ despite clear data that the claudin-2 pore is exquisitely size selective and cannot accommodate large macromolecules. ${ }^{93,108}$ This reflects the limited appreciation of pore, leak, and unrestricted pathways, as well as the lack of suitable assays to measure flux across these pathways in vivo. We can, hopefully, look forward to the resolution of both of these obstacles as understanding becomes more widespread and technological advances provide probes of different charges and sizes that make it possible to distinguish between these permeability routes in vivo, both in experimental and clinical settings. This is critical, as different therapies will be required to limit the intestinal permeability increases or restore the barrier function, depending on the underlying pathogenic mechanism.

\section{Intestinal epithelial damage in IBD and GVHD}

Epithelial damage increases intestinal permeability via the unrestricted pathway and is an established disease mechanism in both IBD and GVHD. Early experiments that directly tested the role of intestinal epithelial damage in vivo relied on chemically induced injury of the epithelium in rodents with agents such as dextran sodium sulfate. ${ }^{109,110}$ These studies established that intestinal epithelial damage could induce colitis. Consistent with this, chimeric mice expressing dominant negative $\mathrm{N}$-cadherin, which disrupts epithelial differentiation, adhesion, and, likely, barrier function, resulted in local inflammation. ${ }^{111,112}$ However, simple comparison of the morphology of these experimental models to that of the human disease indicates that massive epithelial injury is unlikely to be a mechanism of disease initiation in human IBD. ${ }^{26}$ Since then, mouse models of IBD that more closely mimic human disease, including $\mathrm{IL}^{-10^{-1-}-} \mathrm{TNF}^{\mathrm{ARE}}$, and $\mathrm{CD} 45 \mathrm{RB}^{\mathrm{hi}}$-adoptive transfer have been developed. ${ }^{113-116}$ Each of these includes a component of barrier loss and epithelial damage, but the contributions of these to disease pathogenesis is not entirely clear. $^{117,118}$ Further, the mechanisms of intestinal barrier loss likely vary between models. For example, barrier loss in IL-10 $0^{-1-}$ mice precedes onset of clinically evident disease, is unlikely to reflect epithelial damage, i.e., the unrestricted pathway, and probably reflects increased leak pathway flux initiated by cytokine signaling. Although barrier loss and disease onset are nearly simultaneous in $\mathrm{TNF}^{\triangle \mathrm{ARE}}$ mice, epithelial apoptosis again does not appear to be an initiating 
mechanism and early barrier loss is likely a result of the increased leak pathway flux. These temporal distinctions are likely similar to the clinical situation, wherein increased intestinal permeability is not always present and it has been difficult to identify a single common etiology. One exception to this occurs in a subset of healthy relatives of Crohn's disease patients, where specific NOD-2 polymorphisms are associated with increased intestinal permeability. ${ }^{10,65}$

In contrast to IBD, the etiology of the initiating epithelial damage in GVHD is clear: it is caused by pre-transplant conditioning, i.e., irradiation and chemotherapy. Thus, peritransplant intestinal barrier loss reflects increased flux across the unrestricted pathway. As discussed below, this increased permeability is thought to have a critical role in both establishing the intestine as a target organ and in promoting ongoing systemic disease. Studies in preclinical BMT models, as well as human patients, have revealed a positive correlation between the extent of barrier loss and overall disease severity. ${ }^{12,15,53,54}$ Consistent with this, both lower-intensity conditioning $^{119}$ and treatment with growth factors that promote epithelial repair ${ }^{44,51}$ have been associated with reduced-GVHD severity. Although these observations are compelling, it is important to recognize that reduced intensity conditioning and growth factor therapy have many effects, and that nearly all the data linking intestinal barrier function to GVHD are correlative. The results could, therefore, be explained on the premise that more severe disease results in a greater intestinal damage and barrier loss. Further, given that conditioning is required prior to transplant to permit donor-cell engraftment, it has not been possible to define the role of initial tissue damage in GVHD pathogenesis.

In order to eliminate the conditioning-associated intestinal damage prior to BMT, we recently developed a model using immunodeficient recipients. ${ }^{18}$ This allowed donor immunecell engraftment in the absence of irradiation or chemotherapy. Surprisingly, we found that intestinal damage was not required for GVHD pathogenesis when there was a major histocompatability complex (MHC)-mismatch between donor and recipient-in this setting, GVHD could be initiated without any preceding intestinal damage. In contrast, intestinal damage was required for the development of GVHD when donor and host were MHC-matched, which corresponds to the typical clinical scenario with HLA-matched donor and recipient. ${ }^{18}$ However, although the intestinal damage was necessary, it was not sufficient to induce MHC-matched GVHD. As discussed below, both intestinal damage and inactivation of recipient natural killer (NK) cell cytolytic function were required for MHC-matched GVHD to occur.

We explored the role of intestinal damage and increased permeability in GVHD initiation in several ways. First, we found that intestinal damage, even with NK cell depletion, was insufficient to promote GVHD in antibiotic-treated mice. ${ }^{18}$ Conversely, a single intraperitoneal dose of LPS was sufficient to induce MHC-matched GVHD in NK cell-depleted mice despite the absence of intestinal damage and barrier loss at the time of disease initiation. ${ }^{18}$ Although a similar role for LPS has not been demonstrated in IBD, it is notable that most IBD models require the presence of intestinal microorganisms and, in many cases, can be suppressed by broad-spectrum antibiotics. ${ }^{120-123}$ These and other data support a model where a key contribution of intestinal barrier loss is that it allows an influx of microbial products, e.g., LPS. We have hypothesized that this contributes to the development of a pro-inflammatory cytokine environment that promotes immune activation, further barrier loss, and disease progression.

\section{Tight junction-mediated barrier loss in IBD and GVHD}

In the examples discussed above, early barrier loss in GVHD reflected increased unrestricted pathway flux, whereas that in IBD was likely a result of increased tight junction leak pathway permeability. Although increased leak pathway permeability cannot initiate experimental IBD in immunocompetent mice, ${ }^{124}$ it is sufficient to support MHC-matched GVHD of limited severity in immunodeficient NK celldepleted recipients (Nalle et al., unpublished observations). These data suggest that either leak or unrestricted pathway barrier loss can trigger activation of mucosal immune cells that act to prime a systemic immune response and initiate disease. The data also suggest, however, that disease progression associated with barrier loss may be limited in the context of intact immunoregulation.

MLCK is a well-characterized mediator of tight junctionmediated barrier loss in response to the physiological and pathophysiological stimuli. ${ }^{97,105,124-126}$ Three separate proteins are encoded by the MLCK gene (MYLK); "short" MLCK, "long" MLCK, and telokin. ${ }^{127,128}$ Long MLCK is the isoform expressed in intestinal epithelium and is essential for acute, TNF-induced tight junction-dependent intestinal barrier loss. ${ }^{97,129}$ MLCK-mediated phosphorylation of myosin II regulatory light chain leads to an increase in leak pathway flux. Importantly, TNF, IL-1 $\beta$, and the TNF superfamily member LIGHT (lymphotoxin-like inducible protein that competes with glycoprotein D for HVEM on T-cells) which have all been linked to IBD, increase MLCK expression and activity. ${ }^{99,100,130,131}$ It is, therefore, not surprising that colonic biopsy samples from IBD patients reveal a correlation between disease activity and epithelial MLCK expression and activity. ${ }^{126}$ Although MLCK inhibition has not yet been attempted in patients, long MLCK-deficient mice have delayed barrier loss and attenuated disease severity in adoptive transfer colitis. ${ }^{105}$ TNF, LIGHT, and IL-1 $\beta$ have also been in implicated in GVHD pathogenesis. ${ }^{36,38,132-135}$ Further, our preliminary data suggest that MLCK expression and activity in human small-intestinal epithelium correlates with GVHD severity (Nalle et al., unpublished observations). We have also observed that long MLCK-deficient mice have reduced MHC-matched GVHD severity at late stages of disease. These data suggest that tight junction-mediated MLCK-dependent leak pathway permeability increases are a critical factor in the maintenance of pathogenic activity, as well as progression long after disease initiation. These findings also suggest that approaches to specifically limit intestinal barrier loss may be able to reduce 
GVHD severity with fewer side effects than currently available therapies.

\section{NK cells and barrier loss in IBD and GVHD}

Thus far, our epithelial barrier-centric view has not examined which immune cells influence the barrier and, conversely, are affected by barrier loss. One immune-cell type that deserves special consideration here is the NK cell, owing to recent advances in the understanding of how these cells may have an unexpected regulatory role in IBD and GVHD. ${ }^{18,136-139}$ "Conventional" NK cells are innate lymphocytes that can mediate immune responses through direct killing of target cells or indirectly by secretion of a variety of cytokines, most notably interferon- $\gamma \cdot{ }^{140}$ Although NK cells have long been considered pro-inflammatory, it now appears that they can dampen immune activation in preclinical models of IBD and GVHD. This has generated interest in determining if preservation of NK cell numbers or development of means to augment their regulatory functions might be therapeutic in IBD or GVHD. ${ }^{141-143}$

NK cells have been studied extensively in the context of transplantation, as seminal work over 25 years ago demonstrated a role of NK cells in rejecting the MHC-mismatched transplants. ${ }^{144,145}$ However, it was only recently demonstrated that MHC-mismatched donor NK cells could effectively carry out a graft-versus-leukemia response while causing very little GVHD. ${ }^{146}$ The initial explanation for the NK cell-mediated reduction in GVHD was direct killing of host-derived antigenpresenting cells. ${ }^{146}$ Subsequently, it was established that donor ${ }^{139}$ or recipient NK cells ${ }^{18,136}$ are also important for controlling donor T-cell expansion and target organ infiltration. ${ }^{18}$ Both results are consistent with NK cell-mediated regulation of activated, MHC-matched T-cells, as has been observed in colitis and viral infection models. ${ }^{137,147-150}$ However, the mechanism by which NK cells target activated $\mathrm{T}$-cells is still unclear. The signal could come from the T-cells themselves, as activated T-cells are known to upregulate ligands for the NK cell receptor NKG2D, such as members of the Rae1 family. ${ }^{151,152}$ A non-mutually exclusive explanation is that NK cell cytoxicity is increased in response to the cytokine milieu after BMT. Interestingly, a recent study showed that a recombinant TLR-5 agonist could increase NK cell cytoxicity in a viral model, ${ }^{153}$ which could help explain a previous observation that peri-transplant administration of the bacterial product and TLR-5 agonist flagellin can reduce the severity of experimental GVHD. ${ }^{57}$ It therefore appears that there is an underappreciated link between NK cell activation, barrier loss, and GVHD that merits further investigation.

The relevance of NK cells to IBD pathogenesis has not been clearly defined. In dextran sodium sulfate colitis, antibodymediated NK cell depletion leads to more severe inflammation and markedly decreased survival. ${ }^{138}$ This may be because NK cell depletion increased neutrophil infiltration into the inflamed colon. Although epithelial barrier function was not examined, it is likely that the enhanced neutrophil recruitment was secondary to heighted translocation of microbial products owing to epithelial damage and increased flux across the unrestricted pathway. Two other studies using adoptive transfer colitis models have also shown that NK cells can inhibit the proliferation and activation of $\mathrm{CD} 4{ }^{+} \mathrm{T}$-cells, although as above, neither study examined epithelial barrier function. ${ }^{137,149}$ Recently, some of the immunoregulatory effects of NK cells in both the intestine, and the lung, have been attributed to IL-22 production. ${ }^{154-158}$ This may provide an underlying mechanism for some beneficial NK cell effects, as IL-22 has also been implicated in epithelial barrier homeostasis, ${ }^{159}$ and exogenous application of IL-22 is currently being evaluated as a means to treat several diseases, including IBD, GVHD, and psoriasis. ${ }^{160}$

\section{EMERGING THERAPIES AND FUTURE DIRECTIONS}

Current mainstay GVHD therapies focus on broad immunosuppression, which can affect engraftment and, in some contexts, reduce the desired graft-versus-tumor activity. There is, therefore, a need for improved therapeutics that target specific mechanisms in GVHD pathogenesis. Given the accumulation of data that highlight a critical role for the intestinal epithelial barrier in GVHD, it stands that treatments aimed at reducing barrier loss, or alternatively, promoting epithelial healing, could be an effective therapeutic approach. Further, the overlap beteween GVHD and IBD in terms of pathogenic mechanisms suggest that targeted treatments that have been successful for one could be applied to the other. For example, anti-TNF is now common for the treatment of IBD, and is currently being evaluated for efficacy in GVHD. In addition, tacrolimus, which is used in GVHD therapy, also has utility in IBD. Other emerging therapies in IBD and GVHD include modulating cytokine signaling, lymphocyte trafficking, epithelial barrier function, or NK cell activity.

\section{Modulating cytokine signaling or lymphocyte trafficking}

IL-22 is an unusual cytokine in that the IL-22 receptor is not expressed by immune cells. Although T-cells, innate lymphoid cells (ILCs), and NK cells are the major producers of IL-22, IL22 receptor expression is primarily restricted to epithelial cells and fibroblasts and it is through these cells that IL-22 acts to maintain homeostasis of tissue barriers in the intestine, skin, and lung. In a variety of preclinical models, it has been demonstrated that IL-22 signaling is important for promoting wound healing and epithelial regeneration. ${ }^{161,162}$ In particular, IL-22 produced by recipient cells is protective in adoptive transfer colitis and after allogeneic BMT. ${ }^{17,154,163}$ Thus, it has been proposed that exogenous IL-22 could help to restore intestinal barrier function in IBD and GVHD and thereby reduce the overall disease activity. ${ }^{160}$ However, the effect of IL-22 on epithelial barrier function has not been studied in detail.

Barrier restoration has been attempted using KGF, epidermal growth factor, and R-spondin, all of which can be expected to accelerate mucosal healing. ${ }^{44,48,49,51,85,164-168}$ In several studies with GVHD patients, the use of KGF led to a decrease in oral mucositis, but no reduction in the intestinal damage or overall GVHD. ${ }^{46,47,169}$ In one large multicenter study of 
patients with ulcerative colitis, KGF application was safe and well tolerated but failed to show efficacy in inducing remission. ${ }^{170}$ In both instances with human patients, it may be that KGF induces insufficient intestinal epithelial repair. Alternatively, barrier restoration alone may be insufficient to counteract a strong inflammatory response, and application in conjunction with some form of immunomodulation may be necessary.

The integrin $\alpha 4 \beta 7$ on lymphocytes mediates trafficking to the intestine through interaction with the MAdCAM-1 (mucosal addressin cell adhesion molecule-1). There is a strong correlation between $\alpha 4 \beta 7$ expression, accumulation of lymphocytes in the intestine, and inflammation. ${ }^{171}$ Recently, there have been a number of encouraging reports in patients that blocking $\alpha 4 \beta 7$ with the antibody vedolizumab is effective in the induction and maintenance of remission in active IBD,${ }^{172-174}$ and these have led to FDA approval. Whether vedolizumab will realize this potential in larger populations outside of the investigational setting remains to be determined. Mouse models of GVHD also support the view that $\alpha 4 \beta 7$ plays a critical role in regulating intestinal inflammation and overall disease. Specifically, donor T-cells that lack $\alpha 4 \beta 7$ are defective in homing to the intestine after BMT and cause significantly less disease, ${ }^{175}$ and administration of antiMAdCAM-1 antibody decreases GVHD. ${ }^{176}$ In T-cells from patients undergoing allogeneic BMT, the upregulation of $\alpha 4 \beta 7$ correlated with the development of intestinal GVHD. ${ }^{177}$ On the basis of shared pathogenic mechanisms between IBD and GVHD, it is possible that vedolizumab or other $\alpha 4 \beta 7$ blocking antibodies will help reduce intestinal GVHD and overall disease in patients.

\section{Restoring the epithelial barrier}

The appeal of targeted modulation of epithelial tight junction function is ostensibly that disease can be treated with fewer side effects than currently available immunosuppressive and immunomodulatory therapies. Models of acute intestinal inflammation and analyses of knockout mice have been instrumental in identifying the potential pharmacological targets. One exciting candidate whose targeted inhibition could improve the epithelial barrier function is MLCK. In models of acute, cytokine-driven diarrhea, pharmacological or genetic MLCK inhibition prevented barrier loss and diarrhea. ${ }^{98,125}$ These interventions also prevented internalization of the tight junction protein occludin. In vitro data suggest that occludin removal from the tight junction results in increased leak pathway permeability. ${ }^{97,101,178}$ Consistent with this, mice that overexpress occludin within the intestinal epithelium are partially protected from barrier loss and completely protected from diarrhea following TNF administration, ${ }^{101}$ suggesting that preventing occludin internalization may be a viable alternative to MLCK inhibition. A third potential tight junction target is claudin-2, whose expression enhances pore pathway flux. ${ }^{93,94,103}$ Although untested, it remains possible that a specific claudin-2 inhibitor could prevent intestinal barrier loss and limit progression of IBD or GVHD.
Toxicity is a concern with each of these tight junction targets. In particular, it is important to recognize that tight junction barrier function is physiologically regulated during intestinal absorption of water and nutrients, and that inhibition may lead to substantial gastrointestinal complications. Perhaps in part owing to this concern, pharmacological inhibition of tight junction dysregulation in IBD and GVHD has not been reported. Further studies will be needed to identify the optimal targets, delivery systems, and dosing regimens.

\section{NK cell transfer}

Given the emerging regulatory role of NK cells in IBD and GVHD, it is possible that NK cell transfer could be an effective therapeutic approach in both diseases. The feasibility of purifying large numbers of NK cells, either by direct isolation or ex vivo expansion, has been demonstrated. ${ }^{141,179}$ Several very important questions related to this therapeutic approach remain unanswered. Conceptually, although it has been demonstrated that the absence of NK cells results in more severe inflammation and disease in models of IBD and GVHD, the converse, that the presence or addition of NK cells will result in reduced disease, is still unknown. Another more practical question relates to timing and dosing of NK cells. Finally, NK cells from the blood are not homogenous, and include a variety of specialized subsets. ${ }^{180}$ Thus, purification of an empirically defined NK cell "regulatory" subset may yield the most effective results in the context of IBD and GVHD treatment.

\section{CONCLUSIONS}

Advances that define immunoregulatory processes, mechanisms of intestinal barrier loss, and genetic associations in IBD and GVHD have shown us that these diseases have more in common than was previously thought. As a result, it is possible to envision a spectrum of novel therapeutic approaches that have significant advantages over current treatments for IBD and GVHD and do not rely on broad immunosuppression. Both fundamental and practical questions must be addressed if these therapies are to reach patients. Novel approaches, including methods to reduce tight junction leak and pore pathway permeability, as well as means to transfer or expand NK cells and activate their immunoregulatory functions, will need to be developed. The development of effective approaches using epithelial growth factors to promote healing and seal the unrestricted pathway may only require improved delivery vehicles or formulations, and may, therefore, be an accessible therapeutic goal. However, the potential of growth factors to stimulate growth or development of neoplasia cannot be disregarded. In addition, it will be important to consider mechanisms of barrier loss. Use of preclinical models that closely resemble the human pathologies, including MHCmatched BMT for GVHD, and naive T-cell adoptive transfer or genetically modified mice for IBD, will be essential. Finally, just as the development of anti-TNF agents for treatment of rheumatoid arthritis was a great boon to IBD patients, it will be critical to evaluate the ability of new IBD treatments to benefit GVHD patients, and vice versa. Given the similarities between 
IBD and GVHD in terms of genetic links, clinical manifestations, and pathogenic mechanisms, there is a hope that novel treatment approaches will be applicable, and potentially successful, in both diseases.

\section{ACKNOWLEDGMENTS}

This work was supported by National Institute of Health Grants R01DK61931, R01DK68271, R56DK094954, the Crohn's and Colitis Foundation of America, and the Broad Medical Research Foundation.

\section{DISCLOSURE}

The authors declared no conflict of interest.

c 2015 Society for Mucosal Immunology

\section{REFERENCES}

1. Pelaseyed, T. et al. The mucus and mucins of the goblet cells and enterocytes provide the first defense line of the gastrointestinal tract and interact with the immune system. Immunol. Rev. 260, 8-20 (2014).

2. Kostic, A.D., Xavier, R.J. \& Gevers, D. The microbiome in inflammatory bowel disease: current status and the future ahead. Gastroenterology 146, 1489-1499 (2014).

3. Ursell, L.K. et al. The intestinal metabolome: an intersection between microbiota and host. Gastroenterology 146, 1470-1476 (2014).

4. Surana, N.K. \& Kasper, D.L. Deciphering the tete-a-tete between the microbiota and the immune system. J. Clin. Invest. 124, 4197-4203 (2014).

5. Elinav, E., Henao-Mejia, J. \& Flavell, R.A. Integrative inflammasome activity in the regulation of intestinal mucosal immune responses. Mucosal Immunol. 6, 4-13 (2013).

6. Clevers, H.C. \& Bevins, C.L. Paneth cells: maestros of the small intestinal crypts. Annu. Rev. Physiol. 75, 289-311 (2013).

7. Cario, E. Microbiota and innate immunity in intestinal inflammation and neoplasia. Curr. Opin. Gastroenterol. 29, 85-91 (2013).

8. Manichanh, C., Borruel, N., Casellas, F. \& Guarner, F. The gut microbiota in IBD. Nat. Rev. Gastroenterol. Hepatol. 9, 599-608 (2012).

9. Hooper, L.V., Littman, D.R. \& Macpherson, A.J. Interactions between the microbiota and the immune system. Science 336, 1268-1273 (2012).

10. Hollander, D. et al. Increased intestinal permeability in patients with Crohn's disease and their relatives. A possible etiologic factor. Ann. Intern. Med. 105, 883-885 (1986).

11. Odenwald, M.A. \& Turner, J.R. Intestinal permeability defects: is it time to treat? Clin. Gastroenterol. Hepatol. 11, 1075-1083 (2013).

12. Hill, G.R. \& Ferrara, J.L. The primacy of the gastrointestinal tract as a target organ of acute graft-versus-host disease: rationale for the use of cytokine shields in allogeneic bone marrow transplantation. Blood 95, 2754-2759 (2000).

13. Penack, O., Holler, E. \& van den Brink, M.R. Graft-versus-host disease: regulation by microbe-associated molecules and innate immune receptors. Blood 115, 1865-1872 (2010).

14. Nalle, S.C. \& Turner, J.R. Endothelial and epithelial barriers in graft-versushost disease. Adv. Exp. Med. Biol. 763, 105-131 (2012).

15. Johansson, J.E. \& Ekman, T. Gut toxicity during hemopoietic stem cell transplantation may predict acute graft-versus-host disease severity in patients. Dig. Dis. Sci. 52, 2340-2345 (2007).

16. Noth, R. et al. Increased intestinal permeability and tight junction disruption by altered expression and localization of occludin in a murine graft versus host disease model. BMC Gastroenterol. 11, 109 (2011).

17. Hanash, A.M. et al. Interleukin-22 protects intestinal stem cells from immune-mediated tissue damage and regulates sensitivity to graft versus host disease. Immunity 37, 339-350 (2012).

18. Nalle, S.C. et al. Recipient NK cell inactivation and intestinal barrier loss are required for MHC-matched graft-versus-host disease. Sci. Transl. Med. 6, $243 \mathrm{ra} 87$ (2014).

19. Heimesaat, M.M. et al. MyD88/TLR9 mediated immunopathology and gut microbiota dynamics in a novel murine model of intestinal graft-versus-host disease. Gut 59, 1079-1087 (2010).
20. Jenq, R.R. et al. Regulation of intestinal inflammation by microbiota following allogeneic bone marrow transplantation. J. Exp. Med. 209, 903-911 (2012).

21. Eriguchi, Y. et al. Graft-versus-host disease disrupts intestinal microbial ecology by inhibiting Paneth cell production of alpha-defensins. Blood 120, 223-231 (2012).

22. Heidegger, S., van den Brink, M.R., Haas, T. \& Poeck, H. The role of pattern-recognition receptors in graft-versus-host disease and graft-versus-leukemia after allogeneic stem cell transplantation. Front. Immunol. 5, 337 (2014).

23. Schwab, L. et al. Neutrophil granulocytes recruited upon translocation of intestinal bacteria enhance graft-versus-host disease via tissue damage. Nat. Med. 20, 648-654 (2014).

24. Koboziev, I., Karlsson, F., Zhang, S. \& Grisham, M.B. Pharmacological intervention studies using mouse models of the inflammatory bowel diseases: translating preclinical data into new drug therapies. Inflamm. Bowel Dis. 17, 1229-1245 (2011).

25. Ostanin, D.V. et al. T cell transfer model of chronic colitis: concepts, considerations, and tricks of the trade. Am. J. Physiol. Gastrointest. Liver Physiol. 296, G135-G146 (2009).

26. Turner, J.R. in Robbins and Cotran Pathologic Basis of Disease. ed. (Kumar, V. \& Fausto, A. \& Aster, J.814. Saunders Elsevier, Philadelphia, PA, USA, 2010).

27. Ferrara, J.L. Novel strategies for the treatment and diagnosis of graft-versus-host-disease. Best. Pract. Res. Clin. Haematol. 20, 91-97 (2007).

28. Jacobson, P., Uberti, J., Davis, W. \& Ratanatharathorn, V. Tacrolimus: a new agent for the prevention of graft-versus-host disease in hematopoietic stem cell transplantation. Bone Marrow Transplant. 22, 217-225 (1998).

29. Nash, R.A. et al. Phase 3 study comparing methotrexate and tacrolimus with methotrexate and cyclosporine for prophylaxis of acute graft-versushost disease after marrow transplantation from unrelated donors. Blood 96, 2062-2068 (2000).

30. Ng, S.C. \& Kamm, M.A. Therapeutic strategies for the management of ulcerative colitis. Inflamm. Bowel Dis. 15, 935-950 (2009).

31. Baumgart, D.C., Pintoffl, J.P., Sturm, A., Wiedenmann, B. \& Dignass, A.U. Tacrolimus is safe and effective in patients with severe steroid-refractory or steroid-dependent inflammatory bowel disease-a long-term follow-up. Am. J. Gastroenterol. 101, 1048-1056 (2006).

32. Choi, S.W. \& Reddy, P. Current and emerging strategies for the prevention of graft-versus-host disease. Nat. Rev. Clin. Oncol. 11, 536-547 (2014).

33. Ngo, B. et al. Tumor necrosis factor blockade for treatment of inflammatory bowel disease: efficacy and safety. Curr. Mol. Pharmacol. 3, 145-152 (2010).

34. Kobayashi, T. \& Hibi, T. Ulcerative colitis. Which makes patients happier, surgery or anti-TNF therapy? Nat. Rev. Gastroenterol. Hepatol. 11, 272-273 (2014).

35. Mozaffari, S., Nikfar, S., Abdolghaffari, A.H. \& Abdollahi, M. New biologic therapeutics for ulcerative colitis and Crohn's disease. Expert Opin. Biol. Ther. 14, 583-600 (2014).

36. Brown, G.R., Lee, E. \& Thiele, D.L. TNF-TNFR2 interactions are critical for the development of intestinal graft-versus-host disease in MHC class IIdisparate (C57BL/6J->C57BL/6J x bm12)F1 mice. J. Immunol. 168, 3065-3071 (2002).

37. Korngold, R., Marini, J.C., de Baca, M.E., Murphy, G.F. \& Giles-Komar, J. Role of tumor necrosis factor-alpha in graft-versus-host disease and graft-versus-leukemia responses. Biol. Blood Marrow Transplant. 9, 292303 (2003).

38. Hill, G. R. et al. Differential roles of IL-1 and TNF-alpha on graft-versus-host disease and graft versus leukemia. J. Clin. Invest. 104, 459-467 (1999).

39. Busca, A., Locatelli, F., Marmont, F., Ceretto, C. \& Falda, M. Recombinant human soluble tumor necrosis factor receptor fusion protein as treatment for steroid refractory graft-versus-host disease following allogeneic hematopoietic stem cell transplantation. Am. J. Hematol. 82, 45-52 (2007).

40. Yanik, G.A. et al. The impact of soluble tumor necrosis factor receptor etanercept on the treatment of idiopathic pneumonia syndrome after allogeneic hematopoietic stem cell transplantation. Blood 112, 3073-3081 (2008). 
41. Levine, J.E. et al. Etanercept plus methylprednisolone as initial therapy for acute graft-versus-host disease. Blood 111, 2470-2475 (2008).

42. Couriel, D.R. et al. A phase III study of infliximab and corticosteroids for the initial treatment of acute graft-versus-host disease. Biol. Blood Marrow Transplant. 15, 1555-1562 (2009).

43. Gatza, E. et al. Etanercept plus topical corticosteroids as initial therapy for grade one acute graft-versus-host disease after allogeneic hematopoietic cell transplantation. Biol. Blood Marrow Transplant. 20, 1426-1434 (2014).

44. Krijanovski, O.I. et al. Keratinocyte growth factor separates graft-versusleukemia effects from graft-versus-host disease. Blood 94, 825-831 (1999).

45. Ellison, C.A., Gibson, I.W., Hayglass, K.T. \& Gartner, J.G. Effect of palifermin in a murine model of graft-versus-host disease (GVHD) associated with Th2 cytokine production, autoantibody production, and glomerulonephritis. J. Clin. Immunol. 26, 485-494 (2006).

46. Nasilowska-Adamska, B. et al. The influence of palifermin (Kepivance) on oral mucositis and acute graft versus host disease in patients with hematological diseases undergoing hematopoietic stem cell transplant. Bone Marrow Transplant. 40, 983-988 (2007).

47. Levine, J.E., Blazar, B.R., DeFor, T., Ferrara, J.L. \& Weisdorf, D.J. Longterm follow-up of a phase $1 / / 1$ randomized, placebo-controlled trial of palifermin to prevent graft-versus-host disease (GVHD) after related donor allogeneic hematopoietic cell transplantation (HCT). Biol. Blood Marrow Transplant. 14, 1017-1021 (2008).

48. Yamaoka, T., Frey, M.R., Dise, R.S., Bernard, J.K. \& Polk, D.B. Specific epidermal growth factor receptor autophosphorylation sites promote mouse colon epithelial cell chemotaxis and restitution. Am. J. Physiol. Gastrointest. Liver Physiol. 301, G368-G376 (2011).

49. Dube, P.E. et al. Epidermal growth factor receptor inhibits colitisassociated cancer in mice. J. Clin. Invest. 122, 2780-2792 (2012).

50. Zhao, J. et al. R-spondin1, a novel intestinotrophic mitogen, ameliorates experimental colitis in mice. Gastroenterology 132, 1331-1343 (2007).

51. Takashima, S. etal. The Wnt agonist R-spondin 1 regulates systemic graftversus-host disease by protecting intestinal stem cells. J. Exp. Med. 208 285-294 (2011).

52. Takeuchi, O. \& Akira, S. Pattern recognition receptors and inflammation. Cell 140, 805-820 (2010).

53. Cooke, K.R. et al. Tumor necrosis factor- alpha production to lipopolysaccharide stimulation by donor cells predicts the severity of experimental acute graft-versus-host disease. J. Clin. Invest. 102, 1882-1891 (1998).

54. Cooke, K.R. et al. LPS antagonism reduces graft-versus-host disease and preserves graft-versus-leukemia activity after experimental bone marrow transplantation. J. Clin. Invest. 107, 1581-1589 (2001).

55. Zhao, Y. et al. TLR4 inactivation protects from graft-versus-host disease after allogeneic hematopoietic stem cell transplantation. Cell Mol. Immunol. 10, 165-175 (2013).

56. Calcaterra, C. et al. Critical role of TLR9 in acute graft-versus-host disease. J. Immunol. 181, 6132-6139 (2008).

57. Hossain, M.S. et al. Flagellin, a TLR5 agonist, reduces graft-versushost disease in allogeneic hematopoietic stem cell transplantation recipients while enhancing antiviral immunity. J. Immunol. 187, 5130-5140 (2011)

58. Penack, O. et al. NOD2 regulates hematopoietic cell function during graftversus-host disease. J. Exp. Med. 206, 2101-2110 (2009).

59. Ramanan, D., Tang, M.S., Bowcutt, R., Loke, P. \& Cadwell, K. Bacterial sensor Nod2 prevents inflammation of the small intestine by restricting the expansion of the commensal Bacteroides vulgatus. Immunity 41, 311-324 (2014).

60. Couturier-Maillard, A. et al. NOD2-mediated dysbiosis predisposes mice to transmissible colitis and colorectal cancer. J. Clin. Invest. 123, 700-711 (2013).

61. Lorenz, E. et al. Association of TLR4 mutations and the risk for acute GVHD after HLA-matched-sibling hematopoietic stem cell transplantation. Biol. Blood Marrow Transplant. 7, 384-387 (2001).

62. Elmaagacli, A.H. et al. Mutations in innate immune system NOD2/CARD 15 and TLR-4 (Thr399lle) genes influence the risk for severe acute graftversus-host disease in patients who underwent an allogeneic transplantation. Transplantation 81, 247-254 (2006).
63. Elmaagacli, A.H., Koldehoff, M. \& Beelen, D.W. Improved outcome of hematopoietic SCT in patients with homozygous gene variant of toll-like receptor 9. Bone Marrow Transplant. 44, 295-302 (2009).

64. Elmaagacli, A.H. et al. Toll-like receptor 9, NOD2 and IL23R gene polymorphisms influenced outcome in AML patients transplanted from HLA-identical sibling donors. Bone Marrow Transplant. 46, 702-708 (2011)

65. Buhner, S. et al. Genetic basis for increased intestinal permeability in families with Crohn's disease: role of CARD15 3020insC mutation? Gut 55, 342-347 (2006).

66. Cho, J.H. \& Abraham, C. Inflammatory bowel disease genetics: Nod2. Annu. Rev. Med. 58, 401-416 (2007).

67. Cooney, R. \& Jewell, D. The genetic basis of inflammatory bowel disease. Digest. Dis. 27, 428-442 (2009).

68. Strober, W., Asano, N., Fuss, I., Kitani, A. \& Watanabe, T. Cellular and molecular mechanisms underlying NOD2 risk-associated polymorphisms in Crohn's disease. Immunol. Rev. 260, 249-260 (2014).

69. Holler, E. et al. Both donor and recipient NOD2/CARD15 mutations associate with transplant-related mortality and GvHD following allogeneic stem cell transplantation. Blood 104, 889-894 (2004).

70. Holler, E. et al. Prognostic significance of NOD2/CARD15 variants in HLA-identical sibling hematopoietic stem cell transplantation: effect on long-term outcome is confirmed in 2 independent cohorts and may be modulated by the type of gastrointestinal decontamination. Blood 107, 4189-4193 (2006).

71. van der Velden, W.J. et al. NOD2 polymorphisms predict severe acute graft-versus-host and treatment-related mortality in T-cell-depleted haematopoietic stem cell transplantation. Bone Marrow Transplant. 44, 243-248 (2009).

72. Fukata, M. et al. The myeloid differentiation factor 88 (MyD88) is required for CD4 + Tcell effector function in a murine model of inflammatory bowel disease. J. Immunol. 180, 1886-1894 (2008).

73. Tomita, T. et al. MyD88-dependent pathway in T cells directly modulates the expansion of colitogenic CD4 + Tcells in chronic colitis. J. Immunol. 180, 5291-5299 (2008)

74. Rakoff-Nahoum, S., Hao, L. \& Medzhitov, R. Role of toll-like receptors in spontaneous commensal-dependent colitis. Immunity 25, 319-329 (2006).

75. Petnicki-Ocwieja, T. et al. Nod2 is required for the regulation of commensal microbiota in the intestine. Proc. Natl Acad. Sci. USA 106, 15813-15818 (2009).

76. Mondot, S. et al. Altered gut microbiota composition in immune-impaired Nod2(-/-) mice. Gut 61, 634-635 (2012).

77. Hedl, M., Li, J., Cho, J.H. \& Abraham, C. Chronic stimulation of Nod2 mediates tolerance to bacterial products. Proc. Natl Acad. Sci. USA 104, 19440-19445 (2007).

78. Wu, X., Lahiri, A., Haines, G.K. 3rd, Flavell, R.A. \& Abraham, C. NOD2 regulates CXCR3-dependent CD8 $+\mathrm{T}$ cell accumulation in intestinal tissues with acute injury. J. Immunol. 192, 3409-3418 (2014).

79. Franchimont, D. et al. Deficient host-bacteria interactions in inflammatory bowel disease? The toll-like receptor (TLR)-4 Asp299gly polymorphism is associated with Crohn's disease and ulcerative colitis. Gut 53, 987-992 (2004).

80. Henckaerts, L. et al. Mutations in pattern recognition receptor genes modulate seroreactivity to microbial antigens in patients with inflammatory bowel disease. Gut 56, 1536-1542 (2007)

81. Cario, E. Toll-like receptors in inflammatory bowel diseases: a decade later. Inflamm. Bowel Dis. 16, 1583-1597 (2010).

82. Hampe, J. et al. A genome-wide association scan of nonsynonymous SNPs identifies a susceptibility variant for Crohn disease in ATG16L1. Nat. Genet. 39, 207-211 (2007).

83. Gardet, A. \& Xavier, R.J. Common alleles that influence autophagy and the risk for inflammatory bowel disease. Curr. Opin. Immunol. 24 522-529 (2012).

84. Holler, E., Landfried, K., Meier, J., Hausmann, M. \& Rogler, G. The role of bacteria and pattern recognition receptors in GVHD. Int. J. Inflam. 2010, 814326 (2010).

85. Hubbard-Lucey, V.M. et al. Autophagy gene atg1611 prevents lethal T cell alloreactivity mediated by dendritic cells. Immunity 41, 579-591 (2014). 
86. Shen, L., Weber, C.R., Raleigh, D.R., Yu, D. \& Turner, J.R. Tight junction pore and leak pathways: a dynamic duo. Annu. Rev. Physiol. 73, 283-309 (2011).

87. Kalischuk, L.D., Leggett, F. \& Inglis, G.D. Campylobacter jejuni induces transcytosis of commensal bacteria across the intestinal epithelium through M-like cells. Gut. Pathog. 2, 14 (2010).

88. Pravda, J. Crohn's disease: evidence for involvement of unregulated transcytosis in disease etio-pathogenesis. World J. Gastroenterol. 17, 1416-1426 (2011).

89. Tuma, P. \& Hubbard, A.L. Transcytosis: crossing cellular barriers. Physiol. Rev. 83, 871-932 (2003).

90. Johansen, F.E. \& Kaetzel, C.S. Regulation of the polymeric immunoglobulin receptor and IgA transport: new advances in environmental factors that stimulate plgR expression and its role in mucosal immunity. Mucosal Immunol. 4, 598-602 (2011).

91. Schulz, O. \& Pabst, O. Antigen sampling in the small intestine. Trends Immunol. 34, 155-161 (2013).

92. Farquhar, M.G. \& Palade, G.E. Junctional complexes in various epithelia. J. Cell Biol. 17, 375-412 (1963).

93. Weber, C.R. et al. Epithelial myosin light chain kinase activation induces mucosal interleukin-13 expression to alter tight junction ion selectivity. J. Biol. Chem. 285, 12037-12046 (2010).

94. Heller, F. et al. Interleukin-13 is the key effector Th2 cytokine in ulcerative colitis that affects epithelial tight junctions, apoptosis, and cell restitution. Gastroenterology 129, 550-564 (2005).

95. Jovani, M., Fiorino, G. \& Danese, S. Anti-IL-13 in inflammatory bowel disease: from the bench to the bedside. Curr. Drug Targets 14, 1444-1452 (2013)

96. Jordan, W.J. et al. IL-13 production by donor Tcells is prognostic of acute graft-versus-host disease following unrelated donor stem cell transplantation. Blood 103, 717-724 (2004).

97. Clayburgh, D.R. et al. Epithelial myosin light chain kinase-dependent barrier dysfunction mediates T cell activation-induced diarrhea in vivo. J. Clin. Invest. 115, 2702-2715 (2005).

98. Clayburgh, D.R., Musch, M.W., Leitges, M., Fu, Y.X. \& Turner, J.R. Coordinated epithelial NHE3 inhibition and barrier dysfunction are required for TNF-mediated diarrhea in vivo. J. Clin. Invest. 116, 2682-2694 (2006).

99. Schwarz, B.T. et al. LIGHT signals directly to intestinal epithelia to cause barrier dysfunction via cytoskeletal and endocytic mechanisms. Gastroenterology 132, 2383-2394 (2007).

100. Al-Sadi, R., Ye, D., Dokladny, K. \& Ma, T.Y. Mechanism of IL-1betainduced increase in intestinal epithelial tight junction permeability. J. Immunol. 180, 5653-5661 (2008).

101. Marchiando, A.M. et al. Caveolin-1-dependent occludin endocytosis is required for TNF-induced tight junction regulation in vivo. J. Cell Biol. 189, 111-126 (2010).

102. Zeissig, S. et al. Changes in expression and distribution of claudin 2, 5 and 8 lead to discontinuous tight junctions and barrier dysfunction in active Crohn's disease. Gut 56, 61-72 (2007).

103. Raleigh, D.R. et al. Occludin S408 phosphorylation regulates tight junction protein interactions and barrier function. J. Cell Biol. 193, 565582 (2011)

104. Yu, D. et al. MLCK-dependent exchange and actin binding regiondependent anchoring of ZO-1 regulate tight junction barrier function. Proc. Natl Acad. Sci. USA 107, 8237-8241 (2010).

105. Su, L. et al. TNFR2 activates MLCK-dependent tight junction dysregulation to cause apoptosis-mediated barrier loss and experimental colitis. Gastroenterology 145, 407-415 (2013)

106. Gerlach, K. et al. TH9 cells that express the transcription factor PU.1 drive T cell-mediated colitis via IL-9 receptor signaling in intestinal epithelial cells. Nat. Immunol. 15, 676-686 (2014).

107. Bird, L. T cells: IL-9 breaks down barriers. Nat. Rev. Immunol. 14 432 (2014).

108. Gunzel, D. \& Yu, A.S. Claudins and the modulation of tight junction permeability. Physiol. Rev. 93, 525-569 (2013).

109. Clapper, M.L., Cooper, H.S. \& Chang, W.C. Dextran sulfate sodiuminduced colitis-associated neoplasia: a promising model for the development of chemopreventive interventions. Acta. Pharmacol. Sin. 28, 1450-1459 (2007).
110. Cooper, H.S., Murthy, S.N., Shah, R.S. \& Sedergran, D.J. Clinicopathologic study of dextran sulfate sodium experimental murine colitis. Lab. Invest. 69, 238-249 (1993).

111. Hermiston, M.L. \& Gordon, J.I. Inflammatory bowel disease and adenomas in mice expressing a dominant negative $\mathrm{N}$-cadherin. Science 270, 1203-1207 (1995).

112. Hermiston, M.L. \& Gordon, J.I. In vivo analysis of cadherin function in the mouse intestinal epithelium: essential roles in adhesion, maintenance of differentiation, and regulation of programmed cell death. J. Cell Biol. 129, 489-506 (1995).

113. Leach, M.W., Bean, A.G., Mauze, S., Coffman, R.L. \& Powrie, F. Inflammatory bowel disease in C.B-17 scid mice reconstituted with the CD45RBhigh subset of CD4 + T cells. Am. J. Pathol. 148, 1503-1515 (1996).

114. Rennick, D.M., Fort, M.M. \& Davidson, N.J. Studies with IL-10-/- mice: an overview. J. Leukoc. Biol. 61, 389-396 (1997).

115. Kontoyiannis, D., Pasparakis, M., Pizarro, T.T., Cominelli, F. \& Kollias, G. Impaired on/off regulation of TNF biosynthesis in mice lacking TNF AU-rich elements: implications for joint and gut-associated immunopathologies. Immunity 10, 387-398 (1999).

116. te Velde, A.A. et al. Comparative analysis of colonic gene expression of three experimental colitis models mimicking inflammatory bowel disease. Inflamm. Bowel Dis. 13, 325-330 (2007).

117. Turner, J.R. Intestinal mucosal barrier function in health and disease. Nat. Rev. Immunol. 9, 799-809 (2009).

118. Pastorelli, L., De Salvo, C., Mercado, J.R., Vecchi, M. \& Pizarro, T.T. Central role of the gut epithelial barrier in the pathogenesis of chronic intestinal inflammation: lessons learned from animal models and human genetics. Front. Immunol. 4, 280 (2013).

119. Hill, G.R. et al. Total body irradiation and acute graft-versus-host disease: the role of gastrointestinal damage and inflammatory cytokines. Blood 90, 3204-3213 (1997).

120. Powrie, F., Mauze, S. \& Coffman, R.L. CD4 + T-cells in the regulation of inflammatory responses in the intestine. Res. Immunol. 148, 576-581 (1997).

121. Sellon, R.K. et al. Resident enteric bacteria are necessary for development of spontaneous colitis and immune system activation in interleukin-10-deficient mice. Infect Immun. 66, 5224-5231 (1998).

122. Hoentjen, F. et al. Antibiotics with a selective aerobic or anaerobic spectrum have different therapeutic activities in various regions of the colon in interleukin 10 gene deficient mice. Gut 52, 1721-1727 (2003).

123. Gkouskou, K.K., Deligianni, C., Tsatsanis, C. \& Eliopoulos, A.G. The gut microbiota in mouse models of inflammatory bowel disease. Front Cell Infect Microbiol. 4, 28 (2014).

124. Su, L. et al. Targeted epithelial tight junction dysfunction causes immune activation and contributes to development of experimental colitis. Gastroenterology 136, 551-563 (2009).

125. Zolotarevsky, Y. et al. A membrane-permeant peptide that inhibits MLC kinase restores barrier function in in vitro models of intestinal disease. Gastroenterology 123, 163-172 (2002).

126. Blair, S.A., Kane, S.V., Clayburgh, D.R. \& Turner, J.R. Epithelial myosin light chain kinase expression and activity are upregulated in inflammatory bowel disease. Lab. Invest. 86, 191-201 (2006).

127. Lazar, V. \& Garcia, J.G. A single human myosin light chain kinase gene (MLCK; MYLK). Genomics 57, 256-267 (1999).

128. Kamm, K.E. \& Stull, J.T. Dedicated myosin light chain kinases with diverse cellular functions. J. Biol. Chem. 276, 4527-4530 (2001).

129. Clayburgh, D.R. et al. A differentiation-dependent splice variant of myosin light chain kinase, MLCK1, regulates epithelial tight junction permeability. J. Biol. Chem. 279, 55506-55513 (2004).

130. Graham, W.V. et al. Tumor necrosis factor-induced long myosin light chain kinase transcription is regulated by differentiationdependent signaling events. Characterization of the human long myosin light chain kinase promoter. J. Biol. Chem. 281, 26205-26215 (2006).

131. Cao, M., Wang, P., Sun, C., He, W. \& Wang, F. Amelioration of IFN-gamma and TNF-alpha-induced intestinal epithelial barrier dysfunction by berberine via suppression of MLCK-MLC phosphorylation signaling pathway. PLoS One 8, e61944 (2013). 
132. Cooke, K.R. etal. Tumor necrosis factor-alpha neutralization reduces lung injury after experimental allogeneic bone marrow transplantation. Transplantation 70, 272-279 (2000).

133. Tamada, K. et al. Blockade of LIGHT/LTbeta and CD40 signaling induces allospecific T cell anergy, preventing graft-versus-host disease. J. Clin. Invest. 109, 549-557 (2002).

134. Xu, Y. et al. Selective targeting of the LIGHT-HVEM costimulatory system for the treatment of graft-versus-host disease. Blood 109, 4097-4104 (2007).

135. Jankovic, D. et al. The Nirp3 inflammasome regulates acute graft-versushost disease. J. Exp. Med. 210, 1899-1910 (2013).

136. Noval Rivas, M. et al. NK cell regulation of CD4 T cell-mediated graftversus-host disease. J. Immunol. 184, 6790-6798 (2010).

137. Fort, M.M., Leach, M.W. \& Rennick, D.M. A role for NK cells as regulators of CD4 + Tcells in a transfer model of colitis. J. Immunol. 161, 3256-3261 (1998).

138. Hall, L.J. et al. Natural killer cells protect mice from DSS-induced colitis by regulating neutrophil function via the NKG2A receptor. Mucosal Immunol. 6, 1016-1026 (2013).

139. Olson, J.A. et al. NK cells mediate reduction of GVHD by inhibiting activated, alloreactive T cells while retaining GVT effects. Blood 115, 4293-4301 (2010).

140. Cella, M., Miller, H. \& Song, C. Beyond NK cells: the expanding universe of innate lymphoid cells. Front. Immunol. 5, 282 (2014).

141. Passweg, J.R., Stern, M., Koehl, U., Uharek, L. \& Tichelli, A. Use of natural killer cells in hematopoetic stem cell transplantation. Bone Marrow Transplant. 35, 637-643 (2005).

142. Velardi, A., Ruggeri, L., Alessandro, , Moretta, \& Moretta, L. NK cells: a lesson from mismatched hematopoietic transplantation. Trends Immunol. 23, 438-444 (2002).

143. Blazar, B.R., Murphy, W.J. \& Abedi, M. Advances in graft-versus-host disease biology and therapy. Nat. Rev. Immunol. 12, 443-458 (2012).

144. Murphy, W.J., Kumar, V. \& Bennett, M. Acute rejection of murine bone marrow allografts by natural killer cells and T cells. Differences in kinetics and target antigens recognized. J. Exp. Med. 166, 1499-1509 (1987).

145. Murphy, W.J., Kumar, V. \& Bennett, M. Rejection of bone marrow allografts by mice with severe combined immune deficiency (SCID). Evidence that natural killer cells can mediate the specificity of marrow graft rejection. J. Exp. Med. 165, 1212-1217 (1987).

146. Ruggeri, L. et al. Effectiveness of donor natural killer cell alloreactivity in mismatched hematopoietic transplants. Science 295, 2097-2100 (2002).

147. Waggoner, S.N., Taniguchi, R.T., Mathew, P.A., Kumar, V. \& Welsh, R.M. Absence of mouse 2B4 promotes NK cell-mediated killing of activated CD8 $+T$ cells, leading to prolonged viral persistence and altered pathogenesis. J. Clin. Invest. 120, 1925-1938 (2010).

148. Rabinovich, B.A. etal. Activated, but not resting, Tcells can be recognized and killed by syngeneic NK cells. J. Immunol. 170, 3572-3576 (2003).

149. Yamaji, O. et al. The development of colitogenic CD4(+) T cells is regulated by IL-7 in collaboration with NK cell function in a murine model of colitis. J. Immunol. 188, 2524-2536 (2012).

150. Peppa, D. et al. Up-regulation of a death receptor renders antiviral Tcells susceptible to NK cell-mediated deletion. J. Exp. Med. 210, 99-114 (2013).

151. Diefenbach, A., Jensen, E.R., Jamieson, A.M. \& Raulet, D.H. Rae1 and $\mathrm{H} 60$ ligands of the NKG2D receptor stimulate tumour immunity. Nature 413, 165-171 (2001)

152. Spear, P., Wu, M.R., Sentman, M.L. \& Sentman, C.L. NKG2D ligands as therapeutic targets. Cancer Immun. 13, 8 (2013).

153. Hossain, M.S., Ramachandiran, S., Gewirtz, A.T. \& Waller, E.K. Recombinant TLR5 agonist CBLB502 promotes NK cell-mediated anti-CMV immunity in mice. PLoS One 9, e96165 (2014).

154. Zenewicz, L.A. et al. Innate and adaptive interleukin-22 protects mice from inflammatory bowel disease. Immunity 29, 947-957 (2008).

155. Cella, M. et al. A human natural killer cell subset provides an innate source of IL-22 for mucosal immunity. Nature 457, 722-725 (2009).
156. Colonna, M. Interleukin-22-producing natural killer cells and lymphoid tissue inducer-like cells in mucosal immunity. Immunity 31, 15-23 (2009).

157. Kumar, P., Thakar, M.S., Ouyang, W. \& Malarkannan, S. IL-22 from conventional NK cells is epithelial regenerative and inflammation protective during influenza infection. Mucosal Immunol. 6, 69-82 (2013).

158. Xu, X. et al. Conventional NK cells can produce IL-22 and promote host defense in Klebsiella pneumoniae pneumonia. J. Immunol. 192, 1778-1786 (2014).

159. Aujla, S.J. \& Kolls, J.K. IL-22: a critical mediator in mucosal host defense. J. Mol. Med. 87, 451-454 (2009).

160. Sabat, R., Ouyang, W. \& Wolk, K. Therapeutic opportunities of the IL-22IL-22R1 system. Nat. Rev. Drug Discov. 13, 21-38 (2014).

161. Zenewicz, L.A. \& Flavell, R.A. Recent advances in IL-22 biology. Int. Immunol. 23, 159-163 (2011).

162. Rutz, S., Eidenschenk, C. \& Ouyang, W. IL-22, not simply a Th17 cytokine. Immunol. Rev. 252, 116-132 (2013).

163. Sugimoto, K. et al. IL-22 ameliorates intestinal inflammation in a mouse model of ulcerative colitis. J. Clin. Invest. 118, 534-544 (2008).

164. Zeeh, J.M. et al. Keratinocyte growth factor ameliorates mucosal injury in an experimental model of colitis in rats. Gastroenterology 110 , 1077-1083 (1996).

165. Panoskaltsis-Mortari, A., Lacey, D.L., Vallera, D.A. \& Blazar, B.R. Keratinocyte growth factor administered before conditioning ameliorates graft-versus-host disease after allogeneic bone marrow transplantation in mice. Blood 92, 3960-3967 (1998).

166. Egger, B. et al. Keratinocyte growth factor ameliorates dextran sodium sulfate colitis in mice. Dig. Dis. Sci. 44, 836-844 (1999).

167. Yamaoka, T. et al. Transactivation of EGF receptor and ErbB2 protects intestinal epithelial cells from TNF-induced apoptosis. Proc. Natl Acad. Sci. USA 105, 11772-11777 (2008).

168. Frey, M.R., Hilliard, V.C., Mullane, M.T. \& Polk, D.B. ErbB4 promotes cyclooxygenase-2 expression and cell survival in colon epithelial cells. Lab. Invest. 90, 1415-1424 (2010).

169. Blazar, B.R. et al. Phase $1 / 2$ randomized, placebo-control trial of palifermin to prevent graft-versus-host disease (GVHD) after allogeneic hematopoietic stem cell transplantation (HSCT). Blood 108, 3216-3222 (2006).

170. Sandborn, W.J. et al. Repifermin (keratinocyte growth factor-2) for the treatment of active ulcerative colitis: a randomized, double-blind, placebo-controlled, dose-escalation trial. Aliment Pharmacol. Ther. 17, 1355-1364 (2003).

171. Gorfu, G., Rivera-Nieves, J. \& Ley, K. Role of beta7 integrins in intestinal lymphocyte homing and retention. Curr. Mol. Med. 9, 836-850 (2009).

172. Sandborn, W.J. et al. Vedolizumab as induction and maintenance therapy for Crohn's disease. N. Engl. J. Med. 369, 711-721 (2013).

173. Feagan, B.G. et al. Vedolizumab as induction and maintenance therapy for ulcerative colitis. N. Engl. J. Med. 369, 699-710 (2013).

174. Haddley, K. Vedolizumab for the treatment of inflammatory bowel disease. Drugs Today 50, 309-319 (2014).

175. Petrovic, A. et al. LPAM (alpha 4 beta 7 integrin) is an important homing integrin on alloreactive $T$ cells in the development of intestinal graftversus-host disease. Blood 103, 1542-1547 (2004).

176. Ueha, S. et al. Intervention of MAdCAM-1 or fractalkine alleviates graft-versus-host reaction associated intestinal injury while preserving graft-versus-tumor effects. J. Leukoc. Biol. 81, 176-185 (2007).

177. Chen, Y.B. et al. Up-regulation of alpha4beta7 integrin on peripheral Tcell subsets correlates with the development of acute intestinal graft-versushost disease following allogeneic stem cell transplantation. Biol. Blood Marrow Transplant. 15, 1066-1076 (2009).

178. Van Itallie, C.M., Fanning, A.S., Holmes, J. \& Anderson, J.M. Occludin is required for cytokine-induced regulation of tight junction barriers. J. Cell Sci. 123, 2844-2852 (2010).

179. Chouaib, S. et al. Improving the outcome of leukemia by natural killer cell-based immunotherapeutic strategies. Front. Immunol. 5, 95 (2014).

180. Cooper, M.A., Fehniger, T.A. \& Caligiuri, M.A. The biology of human natural killer-cell subsets. Trends Immunol. 22, 633-640 (2001). 